\title{
FUNCIONAMIENTO FAMILIAR Y SU RELACIÓN CON LA EXPOSICIÓN A LA VIOLENCIA EN ADOLESCENTES MEXICANOS
}

\author{
RELATIONSHIP BETWEEN FAMILY FUNCTIONING AND \\ EXPOSURE TO VIOLENCE AMONG MEXICAN ADOLESCENTS
}

\author{
Julia Gallegos-Guajardo ${ }^{1}$, NORMA A. RuvalCABA-ROMERO ${ }^{2}$, \\ Jesús CASTILLO-LÓPEZ ${ }^{1}$ Y PAUlina C. AyAlA-DíAZ ${ }^{1}$
}

Cómo referenciar este artículo/How to reference this article:

Gallegos-Guajardo, J., Ruvalcaba-Romero, N. A., Castillo-López, J. y Ayala-Díaz, P. C. (2016). Funcionamiento familiar y su relación con la exposición a la violencia en adolescentes mexicanos [Relationship between family functioning and exposure to violence among Mexican adolescents]. Acción Psicológica, 13(2), 69-78. http://dx.doi.org/10.5944/ap.13.2.17810

\section{Resumen}

La presente investigación tuvo como objetivo estudiar la relación entre el funcionamiento familiar y la exposición a la violencia en adolescentes mexicanos. Se propuso un diseño transversal, descriptivo y correlacional. La muestra estuvo conformada por 133 estudiantes de bachillerato con edades entre los 15 a 19 años de edad.
Como instrumentos se utilizaron dos cuestionarios: La Escala de Evaluación de la Cohesión y Adaptabilidad Familiar (FACES IV-Esp) de Rivero, MartínezPampliega y Olson (2010) y el Cuestionario de Exposición a la Violencia (CEV) de Orue y Calvete (2010). Los resultados muestran diferencias de género en cuanto a la observación de la violencia en la calle y la victimización en la escuela y en la calle. Se muestran también diferencias de género en variables del funcionamiento familiar tales como: flexibilidad,

Correspondencia: Julia Gallegos Guajardo. Departamento de Psicología, División de Ciencias de la Salud, Univesidad de Monterrey, México.

Email: gallegosjulia@gmail.com

${ }^{1}$ Universidad de Monterrey, México.

${ }^{2}$ Universidade Guadalajara, México.

Recibido: 23 de enero de 2016.

Aceptado: 05 de abril de 2016. 
cohesión, satisfacción y comunicación familiar. Los resultados del análisis de correlación sugieren que a mayor flexibilidad, cohesión, satisfacción y comunicación familiar menor observación de violencia en la escuela y en la casa, y menor victimización en casa. Se observó también una predicción significativa de la cohesión familiar sobre la victimización en casa. Finalmente, se concluye la importancia de fortalecer los vínculos familiares $\mathrm{y}$ de fomentar un funcionamiento familiar positivo y balanceado. Esto con el objetivo de que la familia ejerza un efecto protector ante la exposición a la violencia.

Palabras clave: funcionamiento familiar; exposición a violencia; adolescentes mexicanos; cohesión familiar; comunicación familiar.

\begin{abstract}
The aim of this study was to investigate the relationship between family functioning and exposure to violence among Mexican adolescents. The design of this study was transversal, descriptive, and correlational. Participants were 133 high school students, ages 15 to 18. Two instruments were administered: La Escala de Evaluación de la Cohesión y Adaptabilidad Familiar, in English The Family Adaptability and Cohesion Evaluation Scale, (FACES IV-Esp) by Rivero, MartínezPampliega and Olson (2010), and Cuestionario de Exposición a la Violencia, in English Exposure to Violence Questionnaire, by Orue and Calvete (2010). Results showed statistically significant gender differences related to observing violence in the streets, and victimization in the school and in the street. Gender differences were also significant in family functioning variables such as: flexibility, cohesion, satisfaction, and family communication. Results from the correlational analysis suggested that the higher the flexibility, cohesion, satisfaction and family communication, the lower the violence observation at school and at home, and the lower victimization at home. Predictive validity was also observed for family cohesion over victimization at home. As a conclusion, it is important to strengthen family ties and to promote a positive and balanced
\end{abstract}

family functioning, with the purpose of families being a protective factor against violence exposure.

Keywords: family functioning; exposure to violence; Mexican adolescents; family cohesion; family communication.

\section{Introducción}

El índice de violencia en México se ha incrementado y su prevención ha sido tema prioritario dentro de la agenda nacional. Diferentes investigaciones han estudiado la exposición a la violencia en diferentes contextos. En un estudio realizado por Medina-Mora (2009) los resultados muestran que el $24.6 \%$ de los participantes había sido asaltado con un arma, el $20.3 \%$ había sido testigo de violencia familiar en su infancia, y el $18.3 \%$ víctima de violencia severa por parte de padres o cuidadores en ese periodo temprano de vida. Aproximadamente el $7.4 \%$ había sido golpeado por una persona ajena a los padres o pareja, $5.4 \%$ había experimentado abuso sexual, y 3.9 \% había sufrido de una violación. Las mujeres presentaron mayor prevalencia de abuso sexual, violencia física por parte de la pareja, violación, persecución y acoso, y los hombres experimentaron más violencia relacionada con armas y accidentes.

En relación al alto índice de violencia en el hogar, otros estudios han reportado resultados similares. Por ejemplo, en un estudio realizado con 5484 estudiantes entre los 14 y 19 años de edad, se encontró que el $7.5 \%$ reportaron ser víctimas de violencia física en el hogar, cerca de $16.5 \%$ de violencia verbal y casi el $2 \%$ haber sido sometidos a abuso sexual (Espinoza et al., 2010).

La Encuesta Nacional de Salud y Nutrición del 2006 estudió la distribución de adolescentes de 10 a 19 años de edad que sufrieron daños por alguna violencia. Los resultados muestran que el $6.8 \%$ fue con armas de fuego, el $8.3 \%$ con objetos cortantes, el $59 \%$ sufrió golpes, patadas, puñetazos, el $4.6 \%$ agresión sexual, y el $21.4 \%$ sufrieron otro tipo de agresiones y maltrato. En todos los tipos de violencia, salvo en agresión sexual, los hombres ocuparon el mayor porcentaje. En relación al lugar en 
donde ocurre la violencia, los resultados muestran que: el $58.9 \%$ ocurre en una vía pública, el $24.7 \%$ en la escuela, el $10.7 \%$ en el hogar, el $2.1 \%$ en el campo, el $1.3 \%$ en lugar de recreo o deportivo, el $1.5 \%$ en un establecimiento comercial, y el $0.8 \%$ en otro tipo de lugares. Las mujeres, a comparación de los hombres, mostraron mayor exposición a la violencia en el hogar. Mientras que los hombres, a comparación de las mujeres, mostraron mayor exposición a la violencia en la calle o en la escuela (Olaiz et al., 2006).

La Primera Encuesta Nacional de Exclusión, Intolerancia y Violencia en Escuelas Públicas de Educación Media Superior, muestra que aproximadamente el $35 \%$ de los estudiantes han sido insultados, el $40 \%$ ignorados, el $35 \%$ rechazados, el $11 \%$ han sido golpeados por sus compañeros, al $28 \%$ le han puesto apodos y al $21 \%$ le han escondido sus pertenencias. Respecto al género, los resultados señalan que el $72 \%$ de los hombres y el $65 \%$ de las mujeres, han estado expuestos a acciones de agresión o violencia (Secretaría de Educación Pública [SEP], 2008).

\section{Funcionamiento Familiar y Exposición a la Violencia}

El ambiente familiar de los jóvenes y sus interacciones pueden afectar su exposición a la violencia a través de diversos mecanismos. Por ejemplo, la violencia familiar modela los comportamientos agresivos en los miembros de la familia, y por otro lado el establecimiento de normas en pro de la tolerancia y la paz, disminuye la aparición de conductas violentas (Baldry, 2003) y favorece la resolución pacífica del conflicto (Ruvalcaba, Murrieta, Rayón y Pimentel, 2015). El monitoreo parental también tiene un rol importante, ya que cuando los jóvenes no son adecuadamente supervisados por sus padres, o por el contrario cuando los padres son excesivamente intrusivos, es más probable la exposición a la violencia, tanto como víctima como perpetrador (Simmons-Morton, Hartos y Haynie, 2004). Otros aspectos como la poca calidez parental, la baja cohesión familiar, el poco involucramiento de los padres de familia, y la estructura familiar, también tienen un impacto en la exposición a la violencia (Flouri y Buchanan, 2003); no obstante, se encuentra un efecto protector ante las conductas violentas cuando la comunicación con el padre es positiva y abierta (Estévez, Murgui, Moreno y Musitu, 2007).

La funcionalidad familiar implica conceptos como: cohesión familiar, estilos parentales democráticos, apego emocional y estrategias de resolución de conflictos (Oljača, Erdeš-Kavečan y Kostović, 2012) y se refiere a la capacidad que tiene la familia de mantener su sistema a pesar de los eventos o amenazas que puedan generar cambios en alguno de sus miembros. Los padres de familia juegan un papel determinante en el proceso de construcción de identidad de sus hijos, y se ha constatado que un ambiente familiar positivo funge como factor protector ante comportamientos problemáticos (Chunga, 2008; Levin, Dallgado y Curie, 2012).

Existen estudios que han explorado la diferencia de género entre adolescentes en la percepción de sus padres. Por ejemplo, en el estudio realizado por Mestre, Samper, Tur y Díez (2010), se pudo concluir un perfil diferencial de las mujeres y los hombres adolescentes, en donde las mujeres informaban percibir más afecto, apoyo emocional y evaluación positiva por parte de los padres y más respeto por su forma de pensar. Y, por el contrario, los hombres percibían más hostilidad y evaluación negativa, castigos e intrusividad por parte de los padres. Complementariamente, en estudio realizado por Murrieta, $\mathrm{Ru}-$ valcaba, Caballo y Lorenzo (2014), se observa que los varones reciben más mensajes de aprobación de la violencia por parte de sus padres que lo percibido por las mujeres.

Algunos estudios han investigado la relación entre la funcionalidad familiar y la exposición a la violencia. Sobre la violencia en la escuela, Spriggs, Lannotti, Nansel y Haynie (2007) realizaron un estudio para explorar la relación que existe entre el bullying, tanto de víctimas como perpetuadores, y los factores familiares, de relación entre pares, y los escolares en adolescentes estadounidenses. En relación al factor familiar, los autores reportan que la relación familiar percibida por los alumnos está asociada con su involucramiento en el bullying. Tanto víctimas como perpetradores reportaron un nivel bajo de involucramiento por parte de sus padres en la es- 
cuela y especialmente estos últimos, manifestaron también una comunicación más difícil con sus padres.

De manera similar, un estudio realizado por Uribe, Orcasita y Aguillón (2012) reportó que un alto número de estudiantes colombianos se han involucrado en el bullying, como víctimas y agresores, y se describe una clara asociación entre la ausencia de vinculación afectiva y apoyo entre la familia y la emisión de conductas de enfrentamiento entre estudiantes. La investigación evidenció una relación importante entre los estudiantes que son agresores y el funcionamiento familiar que perciben, moderadamente o gravemente disfuncional. CabreraFuentes (2011) encontró también una relación significativa en estudiantes de Ecuador, que sugiere que, a menor nivel de funcionalidad familiar, mayor nivel de bullying.

Sobre la relación entre funcionalidad familiar y la exposición a la violencia en el hogar, Almenares-Aleaga, Louro-Bernal y Ortiz-Gómez (1999) estudiaron este fenómeno en 818 familias de Chile. Los resultados muestran el $12 \%$ de las familias, evidenciaban violencia intrafamiliar, y el $56 \%$ de las familias con violencia presentaba problemas en cuanto a su dinámica relacional sistémica, familias con dificultades en la comunicación, cohesión, estructura de poder, y dificultades para vivenciar y demostrar emociones positivas, y para cumplir funciones y responsabilidades negociadas en el núcleo familiar. Datos similares han sido reportados sobre familias cubanas por Duany-Navarro y Ravelo-Pérez (2005), en donde se reportó que en el $31.3 \%$ de las familias disfuncionales, existía violencia.

La relación entre funcionalidad familiar y la exposición a la violencia en la televisión ha sido poco estudiada, sin embargo, autores como Sandoval-Escobar (2006) han indicado que la televisión posee efectos combinados con otros medios de socialización y que, bajo condiciones sociales desfavorables, como podría ser la disfuncionalidad familiar, agudiza el impacto de la violencia y el contexto antisocial de las audiencias.

Este trabajo busca identificar la relación entre el funcionamiento familiar y la exposición a la violencia en los ámbitos de casa, escuela y calle, así como indagar en la posible diferencia de géneros en la percepción de ambas variables.

\section{Método}

La presente investigación es un estudio transversal, descriptivo y correlacional.

\section{Participantes}

La muestra se constituyó por 133 adolescentes, de 15 a 19 años de edad, con una media de 16.5 años. De los participantes, el $67 \%$ eran hombres y el $33 \%$ eran mujeres, todos estudiantes de bachillerato en la ciudad de Monterrey, Nuevo León. La muestra se obtuvo por conveniencia y es parte de la muestra de un estudio previo realizado por Ayala-Díaz et al. (2014).

\section{Instrumentos de evaluación}

\section{Funcionamiento familiar}

Se utilizó la Escala de Evaluación de la Cohesión y Adaptabilidad Familiar: FACES IV-Esp (Rivero, Martínez-Pampliega y Olson, 2010). Se trata de la adaptación al español de la escala FACES-IV (Olson, 2008). Es una escala tipo Likert de 24 ítems que contiene dos sub escalas independientes, una que considera las características de balance de una familia y que incluye los factores centrales del modelo: Cohesión y Adaptabilidad, mientras que la segunda sub escala contiene los cuatro criterios de desajuste familiar y que se refieren a la rigidez, al caos, al desapego y al sobre-involucramiento. En el estudio de validación está escala arrojó un índice de fiabilidad interna de .93 (Olson, 2011).

Cabe señalar que también se aplicaron de la versión original, la subescala relativa a la satisfacción con la familia y a la Comunicación familiar, ambas tipo likert de 5 puntos y con 10 ítems cada una. El índice de fiabilidad en este estudio, fue de $\alpha=.919$ y .925 respectivamente. 
Tabla 1

Resultados descriptivos y análisis de la varianza por género respecto a las variables utilizadas en este estudio

\begin{tabular}{|c|c|c|c|c|c|}
\hline \multirow{2}{*}{ Variables } & \multicolumn{2}{|c|}{ Hombres } & \multicolumn{2}{|c|}{ Mujeres } & \multirow{2}{*}{$\begin{array}{c}\text { ANOVA } \\
F\end{array}$} \\
\hline & $\mathrm{M}$ & DT & $M$ & DT & \\
\hline \multicolumn{6}{|l|}{ Exposición a la violencia } \\
\hline Observación en la escuela & 4.83 & 3.09 & 4.45 & 2.92 & 0.46 \\
\hline Observación en la calle & 6.89 & 2.52 & 5.50 & 2.94 & $8.04^{* *}$ \\
\hline Observación en la casa & 1.20 & 1.92 & 1.59 & 2.16 & 1.13 \\
\hline Observación en la TV & 7.83 & 3.05 & 7.11 & 2.76 & 1.75 \\
\hline Victimización en la escuela & 1.70 & 1.96 & 0.95 & 1.41 & $5.06^{*}$ \\
\hline Victimización en la calle & 2.34 & 2.46 & 1.45 & 2.12 & $4.21^{*}$ \\
\hline Victimización en la casa & 0.64 & 1.46 & 1.07 & 1.87 & 2.06 \\
\hline \multicolumn{6}{|l|}{ Funcionamiento familiar } \\
\hline Adaptabilidad & 14.73 & 2.62 & 13.50 & 3.31 & $5.48^{*}$ \\
\hline Cohesión & 15.28 & 2.32 & 14.07 & 3.78 & $5.22^{*}$ \\
\hline Desapego & 7.28 & 1.95 & 7.36 & 2.08 & 0.05 \\
\hline Sobre involucramiento & 10.18 & 1.60 & 9.89 & 1.74 & 0.93 \\
\hline Caos & 7.36 & 1.84 & 7.66 & 1.84 & 0.78 \\
\hline Rigidez & 8.84 & 1.81 & 8.75 & 2.00 & 0.07 \\
\hline Satisfacción familiar & 37.56 & 7.12 & 31.58 & 8.92 & $17.26^{* *}$ \\
\hline Comunicación familiar & 38.22 & 6.71 & 33.18 & 8.92 & $13.34^{* *}$ \\
\hline
\end{tabular}

Nota. ${ }^{*} p<.05 .{ }^{* *} p<.01$.

\section{Exposición a la violencia}

Se utilizó el cuestionario de Exposición a la violencia (CEV) de Orue y Calvete (2010), que consta de 21 ítems, de los cuales nueve son de exposición directa o victimización y 12 de exposición indirecta, es decir cuando los sujetos son testigos de la violencia. Así mismo este instrumento considera los escenarios posibles en los que un estudiante puede verse expuesto a la violencia: casa, escuela, calle y televisión. El Alfa de Cronbach resultante en este estudio fue de .861 .

\section{Procedimiento}

Se realizó una invitación para participar en el presente estudio a una preparatoria afiliada a una universidad privada del noreste de México. Se obtuvieron de manera oficial los permisos por parte de los directivos de la preparatoria. Posteriormente seis estudiantes, cursando el último año de la Licenciatura en Psicología, asistieron a seis salones de clases con los alumnos que cursaban el segundo año de la preparatoria. Las aplicaciones de instrumentos fueron grupales y supervisadas por un profesor de Psicología, director de tesis del estudio realizado por Ayala-Díaz et al. (2014).

Este estudio fue aprobado por el Comité de Ética de la universidad. Antes de proceder con la aplicación de instrumentos, se les explicó a los alumnos sobre el proyecto de investigación y que su participación en él era totalmente voluntaria y confidencial, obteniendo el consentimiento informado de todos los participantes que conforman la muestra de este estudio. Se obtuvieron los datos descriptivos de la muestra para posteriormente realizar un análisis de correlación y finalmente identificar el nivel predictivo de las variables relativas al funcionamiento familiar sobre la exposición a la violencia a través de análisis de regresión lineal.

\section{Resultados}

La Tabla 1 muestra los resultados para cada uno de los instrumentos utilizados. Al realizar la comparación entre hombres y mujeres, los resultados mostraron significancia estadística. Se destaca que los hombres suelen estar más expuestos a la violencia en los ámbitos de calle 
Tabla 2

Correlaciones entre el funcionamiento familiar y la exposición a la violencia

\begin{tabular}{|c|c|c|c|c|c|c|c|}
\hline & \multicolumn{4}{|c|}{ Observación } & \multicolumn{3}{|c|}{ Victimización } \\
\hline & Escuela & Calle & Casa & TV & Escuela & Calle & Casa \\
\hline Adaptabilidad & $-.17^{*}$ & .11 & -.23 & .11 & .10 & .22 & -.19 \\
\hline Cohesión & $-.23^{\star \pi}$ & -.07 & $-.36^{\pi \pi}$ & .03 & -.05 & .12 & $-.33^{x \times}$ \\
\hline Desapego & .14 & .09 & $.17^{x}$ & .15 & .03 & -.06 & $.24^{\pi \times}$ \\
\hline Sobre involucramiento & -.14 & -.10 & -.13 & -.05 & -.01 & .03 & -.09 \\
\hline Caos & .08 & .16 & -.04 & $.28^{*}$ & .02 & .07 & .01 \\
\hline Rigidez & .02 & .07 & -.01 & .01 & -.03 & -.02 & -.05 \\
\hline Satisfacción familiar & $-.25^{\star \pi}$ & .02 & $-.38^{\pi \pi}$ & -.09 & -.08 & .11 & $-.35^{x \times}$ \\
\hline Comunicación familiar & $-.25^{\pi x}$ & -.05 & $-.34^{\pi \pi}$ & -.08 & -.06 & .07 & $-.34^{x \times 1}-x-10$ \\
\hline
\end{tabular}

Nota. ${ }^{*} p<.05 .{ }^{* *} p<.01$.

y escuela; no así al interior del hogar en donde no existe diferencia significativa entre mujeres y hombres.

Respecto a las variables de funcionamiento familiar, son los hombres quienes perciben una mayor flexibilidad, cohesión y comunicación en sus casas, sintiéndose además más satisfechos con las dinámicas de sus familias.

Con la intención de identificar la relación entre las variables se llevaron a cabo análisis de correlación, cuyos resultados podemos apreciar en la Tabla 2.

Los resultados muestran que existe una relación negativa y significativa en el ámbito doméstico entre la exposición a la violencia y algunas variables del funcionamiento familiar, específicamente con la adaptabilidad, la cohesión, la satisfacción con la familia y la comunicación, mientras que existe una relación positiva con el desapego. Respecto al ámbito escolar, solamente se en- contraron relaciones negativas entre la observación de la violencia, más no en la victimización. Finalmente, se observa que la observación de la violencia en los medios televisivos, está relacionada positivamente con la percepción de caos al interior de la familia.

Dado que la mayoría de las relaciones estadísticamente significativas fueron las que se encontraron entre el funcionamiento familiar y la exposición a la violencia en el ámbito doméstico, se procedió a hacer un análisis de regresión con la variable de victimización en casa como la variable dependiente. Los resultados se muestran en la Tabla 3.

Se puede observar que en el primer modelo el desapego es significativo con un nivel predictivo del $5.7 \%$ sobre la victimización en casa; sin embargo, en el segundo modelo al incluir las variables que pueden ejercer un efecto protector sobre la violencia familiar, como son la cohesión y la flexibilidad, el desapego pierde sig-

Tabla 3

Análisis de regresión para las variables que pronostican la victimización en casa

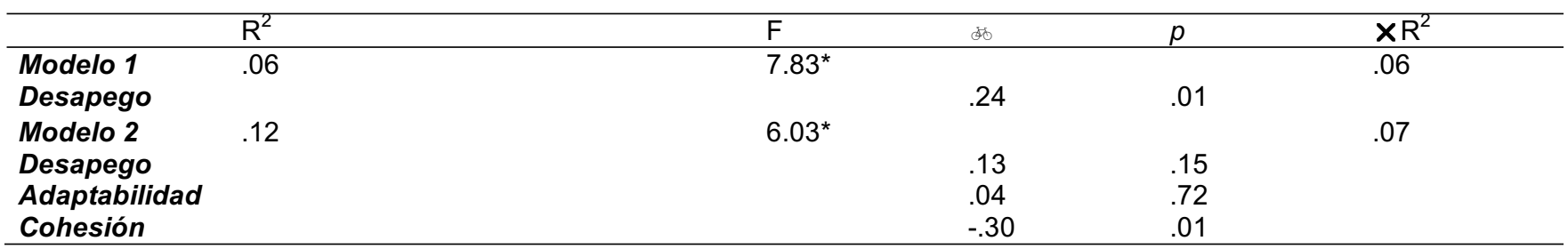

Nota. ${ }^{*} p<.05 .{ }^{* \star} p<.01$. 
nificancia. El modelo total explica en un $12.4 \%$ la victimización en casa, sugiriendo que la cohesión familiar es un factor protector de la violencia en el entorno doméstico.

\section{Discusión}

La familia es el núcleo más importante en el que se desarrolla una persona, es el agente de socialización primario al ser la primera fuente de información del niño acerca de las normas y roles, y de las expectativas que se esperan de él o ella (Cuervo, 2010; Mestre, Samper, Tur y Díez, 2001). El explorar las diferentes variables, como el funcionamiento familiar, que pueden estar relacionadas con la exposición a la violencia es una tarea importante hacia la prevención, porque la exposición a la violencia está también asociada con el abuso de substancias, los problemas psicológicos, y problemas de salud física (Fekkers, Pijpers, Fredriks, Vogels y Verloove-Vanhorick, 2006; Kaltiala-Heino, Rimpela, Rantanen y Rimpela, 2000; Nansel et al., 2001).

La relación entre funcionalidad familiar y exposición a la violencia en casa resultó ser la más fuerte. Los resultados de este estudio que comprueban que a mayor adaptabilidad, cohesión, satisfacción y comunicación familiar, menor observación de violencia y victimización en casa, y concuerdan con investigaciones previas que destacan la influencia recíproca que tienen la funcionalidad familiar y la exposición a la violencia en casa, tanto en el rol de observador como en el de víctima (Almenares-Aleaga, Louro-Bernal y Ortiz-Gómez, 1999; Duany-Navarro y Ravelo-Pérez, 2005).

En relación a las de género, los resultados del presente estudio son similares a previos estudios (Olaiz et al., 2006; SEP, 2008) que muestran que un mayor número de hombres adolescentes, en comparación de mujeres, son expuestos a la violencia, particularmente en la calle y en la escuela. Sin embargo, no se encontraron diferencias estadísticamente significativas respecto a la victimización en el hogar. Un hallazgo inesperado fueron las diferencias de género encontradas en la percepción de la funcionalidad familiar, en donde se encontró que los hombres, en comparación con las mujeres, percibían una mayor adaptabilidad, cohesión, comunicación, y satisfacción familiar. Esto podría explicarse a partir de las diferencias de crianza que culturalmente han permeado a las familias mexicanas, en donde suele existir una mayor flexibilidad con las normas en el caso de los varones. En la cultura mexicana los varones, a comparación de las mujeres, frecuentemente tienen más libertades en cuánto el tiempo que pasan fuera de casa, las personas que frecuentan y las horas de llegada a casa, lo cual incrementa la posibilidad de que estén expuestos con más frecuencia a lugares peligrosos. De igual manera, se ha planteado la influencia del fenómeno de la "masculinidad" en donde los hombres jóvenes muestran un mayor arrojo y atrevimiento ante situaciones de violencia, mientras que las mujeres responden más de manera evasiva (Briseño-León, 2007). Se sugiere indagar más en esta hipótesis.

Diferentes estudios han encontrado que el ser víctima o victimario en el ámbito escolar, está relacionado con la disfuncionalidad familiar, específicamente con el escaso involucramiento de los padres y una difícil comunicación familiar (Cabrera-Fuentes, 2011; Spriggs et al., 2007; Uribe, Orcasita y Aguillon, 2012); no obstante en nuestro estudio encontramos que las dimensiones del funcionamiento familiar están relacionadas con la observación de violencia en la escuela, pero no con la victimización, cabe señalar que al respecto son principalmente las variables positivas las que presentaron las correlaciones estadísticamente significativas, por lo que se afianza la función de la adaptabilidad y la cohesión familiar como factores protectores de la violencia; así mismo esto puede explicar el hecho de que un niño que cuenta con dichos elementos en su casa, no haya normalizado la violencia como un medio para la resolución de los conflictos. Al respecto se sugiere indagar en esta relación dada la alta tasa de incidencia de acoso escolar en el país (Loredo-Abdalá, Perea-Martínez y López-Navarrete, 2008; Olaiz et al, 2006).

Respecto a la exposición de la violencia en los medios televisivos, sugerimos ahondar más su relación con la funcionalidad familiar, ya que la encontrada en este estudio, puede estar explicada como posible consecuencia de un grado adecuado de límites y supervisión por parte de los padres que favorezca que el niño esté expuestos a 
contenidos violentos; no obstante otras perspectivas explican que la disfuncionalidad que vive una familia, moldea el tipo y el grado de violencia que observan los adolescentes en la televisión (Sandoval-Escobar, 2006).

\section{Conclusiones}

Respecto a la exposición de la violencia en las conclusiones de este trabajo, se plasman de acuerdo a los objetivos que se plantearon. En primer lugar, se buscó identificar la relación entre el funcionamiento familiar y la exposición a la violencia. Encontramos que de acuerdo a las variables utilizadas, fueron los elementos balanceados del funcionamiento familiar, es decir la Adaptabilidad y la Cohesión, las que tienen una relación negativa con el hecho de ser víctima o testigo de actos de violencia, específicamente en el ámbito doméstico; por otro lado, también se encontró una relación negativa con la satisfacción y la comunicación familiar.

Es importante destacar que respecto a la variable de exposición a la violencia por observación, el funcionamiento familiar, también parece ejercer un factor protector, se sugiere indagar el efecto, por nuestra parte generamos la hipótesis de que los niños que están satisfechos con su familia, que tienen una adecuada comunicación con sus padres, aunado a un seguimiento de las normas en casa y una percepción de cohesión con el grupo familiar, pueden tener mayor capacidad de observar e identificar las conductas violentas al no considerarlas como un medio para la resolución de los conflictos a diferencia de los niños que experimentan violencia en el hogar.

En lo que se refiere a las diferencias de género, se encontraron como era esperado, que los hombres están más expuestos a la violencia; no obstante también puntuaron más alto en la satisfacción con la familia y la comunicación al interior de la misma, por lo que se sugiere como perspectiva de investigación un abordaje desde la perspectiva sociocultural y de género que pueda explicar este fenómeno.
Así mismo, los hallazgos mostraron que la cohesión familiar es la variable que predice en mayor porcentaje la victimización en casa. Esto sugiere que las estrategias para fomentarla deben de ser uno de los componentes principales de los programas de escuela para padres. Aspectos tales como que los miembros de la familia se sientan unidos, que se apoyen mutuamente durante tiempos difíciles, y que a pesar de sus intereses individuales compartan su tiempo libre, resultaron en el presente estudio fungir como factores que protegen a los adolescentes de ser víctimas de violencia. Para este fin, consideramos también importante que los padres aprendan habilidades la comunicación, cuidado, convivencia familiar, monitoreo y supervisión de los hijos, además de tener expectativas claras del desarrollo de los mismos.

Se sugiere que futuras investigaciones continúen en el estudio de este tema, incluyendo a múltiples informantes, realizando mediciones a través del tiempo, e incluyendo a participantes de diferentes zonas, rurales y urbanas. El incluir la variable de estilos parentales sería también muy valioso para comprender a profundidad este fenómeno.

\section{Referencias}

Almenares-Aleaga, M., Louro-Bernal, I. y Ortiz-Gómez, M. T. (1999). Comportamiento de la violencia intrafamiliar. Revista Cubana de Medicina General Integral, 15, 285-292.

Ayala-Díaz, P. C., Cruz-Rodríguez, M., Gracia-González, M. L., Nieto-Manzano, A., Salas-Becerra, E. A. y Velasco-Enríquez, M. F. (2014). Impacto psicosocial en el rendimiento académico de adolescentes de educación (Tesis de licenciatura inédita). Universidad de Monterrey, México.

Baldry, A. C. (2003). Bullying in schools and exposure to domestic violence. Child Abuse and Neglect, 26(7), 713-732. 2134(03)00114-5 
Briceño-León, R. (2007). Sociología de la violencia en América Latina. Ecuador: FLACSO.

Duany-Navarro, A. y Ravelo-Pérez, V. (2005). Violencia intrafamiliar en un área de salud. Revista Cubana de Medicina General Integral, 21, 1-2.

Medina-Mora, M. E., Borges, G., Benjet, C., Lara, M. C., Rojas, E., Fleiz, C., ... y Aguilar-Gaxiola, S. (2009). Estudio de los trastornos mentales en México: Resultados de la encuesta mundial de salud mental. En J. Rodríguez, R. Kohn y S. Aguilar-Gaxiola (Eds.), Epidemiología de los trastornos mentales en América Latina y el Caribe (pp. 90-100). Washington, D.C.: Organización Panamericana de la Salud.

Chunga, L. S. (2008). Niveles de satisfacción familiar y de comunicación entre padres e hijos. Avances en Psicología, 16(1), 119-137.

Cuervo, A. (2010). Pautas de crianza y desarrollo socioafectivo en la infancia. Diversidad de Perspectivas Psicológicas, 6(1), 111-121.

Cabrera-Fuentes, D. M., (2011). Estudio de la correlación múltiple entre el bullying, funcionalidad familiar y bajo rendimiento escolar cognitivo en las asignaturas de lenguaje y matemática. (Tesis de licenciatura inédita). Universidad Central del Ecuador, Facultad de Ciencias Psicológicas.

Espinoza, F., Zepeda, V., Bautista, V., Hernández, C. M., Newton, O. A. y Plasencia, G. R. (2010). Violencia doméstica y riesgo de conducta suicida en universitarios adolescentes. Salud Pública de México, 52(3), 213-219.

Estévez, E., Murgui, S., Moreno, D. y Musitu, G. (2007). Estilos de comunicación familiar, actitud hacia la autoridad institucional y conducta violenta del adolescente en la escuela. Psicothema, 19(1), 108113.
Flouri, E. y Buchanan, A. (2003). The role of mother involvement and father involvement in adolescent bullying behavior. Journal of Interpersonal Violence, 18(6), 4-26. http://dx.doi.org/10.1177/0886260503018006004

Fekkers, M., Pijpers, F. I., Fredriks, A. M., Vogels, T. y Verloove-Vanhorick, S. P. (2006). Do bullied children get ill, or do ill children get bullied? A prospective cohort study on the relationship between bullying and health related symptoms. Pediatrics, 117(5), 15681574. http://dx.doi.org/10.1542/peds.2005-0187

Kaltiala-Heino, R., Rimpela, M., Rantanen, P. y Rimpela, A. (2000). Bullying at school- an indicator of adolescents at risk for mental disorders. Journal of Adolescence, 23(6), 661-674. http://dx.doi.org/10.1006/jado.2000.0351

Medina-Mora, M. E., Borges, G., Benjet, C., Lara, M. C., Rojas, E., Fleiz, C., ... y Aguilar-Gaxiola, S. (2009). Estudio de los trastornos mentales en México: Resultados de la encuesta mundial de la Salud. En J. J. Rodríguez, R. Kohn y S. Aguilar-Gaxiola (Eds.) Epidemiología de los trastornos mentales en América Latina y el Caribe (pp. 79-90). Washington, D.C.: Organización Panamericana de la Salud.

Mestre, M. V., Samper, P., Tur, A. y Díez, I. (2001). Estilos de crianza y desarrollo prosocial de los hijos. Revista de Psicología General y Aplicada, 54(4), 691703.

Nansel, T. R., Overpeck, M., Pilla, R. S., Ruan, W. J., Simons-Morton, B. y Scheidt, P. (2001). Bullying behaviors among US youth: Prevalence association with psychological adjustment. The Journal of the American Medical Association, 258(16), 2094-2100.

Olaiz, F., Rivera, D., Shamah, L., Rojas, R., Villalpando, H. y Hernández, A. (2006). Encuesta Nacional de Salud y Nutrición. Recuperado de http://ensanut.insp.mx/informes/ensanut2006.pdf 
Olson, D. (2008). FACES IV: Manual. Minneapolis, $\mathrm{MN}$ : Life Innovations.

Orue, I. y Calvete, E. (2010). Elaboración y validación de un cuestionario para medir la exposición a la violencia en la infancia y adolescencia. International Journal of Psychology and Psychological Therapy, $10(2), 279-292$.

Olson, D. (2011). FACES IV and the circumplex model: Validation study. Journal of Marital \& Familiy Therapy, 20(1), 64-80. http://dx.doi.org/10.1111/j.1752-0606.2009.00175.x

Oljača, M., Erdeš-Kavečan, Đ. y Kostović, S. (2012). Relationship between the quality of family functioning and the academic achievement of adolescents. Croatian Journal of Education, 14(3), 485-510.

Loredo-Abdalá, A., Perea-Martínez, A. y LópezNavarrete, G. E. (2008). Bullying: Acoso escolar, la violencia entre iguales, problemática real en adolescentes. Acta Pediátrica de México, 29(4), 210214.

Levin, K., Dallago, L. y Currie, C. (2012). The association between adolescent life satisfaction, family structure, family affluence and gender differences in parent-child communication. Social Indicators Research, 106(2), 287-305. http://dx.doi.org/10.1007/s11205-011-9804-y

Murrieta, P., Ruvalcaba, N., Caballo, V. y Lorenzo, M. (2014). Cambios en la percepción de la violencia y el comportamiento agresivo entre niños a partir de un programa de habilidades socioemocionales. Psicología Conductual, 22(3), 569-584.

Rivero, N., Martínez-Pampliega, A. y Olson, D. (2010). Spanish adaptation of the FACES IV questionnaire: Psychometric characteristics. The Family Journal: Counseling and Therapy for Couples and Families, $18(3)$, http://dx.doi.org/10.1177/1066480710372084
Ruvalcaba, N., Murrieta, P., Rayón, J. R. y Pimentel, A. (2015). Aprobación parental de la violencia y victimización como predictores de la agresión y conductas prosociales. Revista Tesis Psicológica, 10(1), 60-71.

Simmons-Morton, B. G., Hartos, J. L. y Haynie, D. L. (2004). Prospective analysis of peer and parent influences on minor aggression among early adolescents. Health Education \& Behavior, 31(19). 22-33. http://dx.doi.org/10.1177/1090198103258850

Sandoval-Escobar, M. (2006). Los efectos de la televisión sobre el comportamiento de las audiencias jóvenes desde la perspectiva de la convergencia y de las prácticas culturales. Universitas Psychologica, 5(2), 205-222.

Spriggs, A. L., Lannotti, R. J., Nansel, T. R. y Haynie, D. L. (2007). Adolescent bullying involvement and perceived family, peer and school relations: Commonalities and differences across race/ethnicity. Journal of Adolescent Health, 41(3), 283-293. http://dx.doi.org/10.1016/j.jadohealth.2007.04.009

Secretaría de Educación Pública (SEP; 2008). Primera Encuesta Nacional de Exclusión, Intolerancia y Violencia en Escuelas públicas de Educación Media Superior. Recuperado de http://catedraunescodh.unam.mx/catedra/BibliotecaV2 /Documentos/Educacion/Informes/SEP_Encuesta_Dis criminacion_y_Violencia_2008.pdf

Uribe, A. F., Orcasita, L. y Aguillón, E. (2012). Bullying, redes de apoyo $y$ funcionamiento familiar en adolescentes de una institución educativa de Santander, Colombia. Psychologia, Avances de la Disciplina, 2, 83-99. 